\title{
THE SPECIAL TYPE FRACTALS INTRODUCTORY CLASSIFICATION
}

\author{
ANATOLY E. VOEVUDKO
}

\begin{abstract}
For the particular selected type of fractals, i.e., the Kronecker product based fractals, the introductory common classification is proposed. It essentially relies on the size and content of the initial fractal matrix. In turn, it gives one of the possible explanation of the phenomenal ability to produce a few of the most popular fractals using many different algorithms, languages and tools. Also, it paves the way to discover on the regular basis thousands of the new fractals, and this gives researchers a wider range of fractal models to choose from.
\end{abstract}

Keywords: Kronecker product, Kronecker power, fractals, classification.

\section{INTRODUCTION}

Introductory common classification of the Kronecker product generated fractals is proposed. This classification essentially relies on the size and content of the initial fractal matrix. The origin, nature and generating technique related to Kronecker product [1] based fractals (KPBF) is explained in more details in [2, 3] A lot of well-known and new fractal samples are shown and KPBF generators are offered both in JavaScript [2] and R 3].

It should be stressed that there is no any attempt to classify KPBF. Each book or article that mentioned KPBF or devoted to it is showing only 1-3 matrix samples and/or related fractal images. See many of such books and articles referenced in [2]. Moreover, many of them are using as a sample mostly already published KPBF.

Here are just a few samples (excluding my own) to support this statement:

- $\mathrm{STF}, \mathrm{SCF}, \mathrm{CBF}$ - in 1 .

- $\mathrm{STF}, \mathrm{SCF}$ - in 4 .

- $\mathrm{STF}, \mathrm{SCF}, \mathrm{CBF}$ - in 5 .

- $\mathrm{HGF}, \mathrm{APF}, \mathrm{APFm}$ - in 6].

- $\mathrm{BF}, \mathrm{XDF}-7$.

- $\mathrm{STF}, \mathrm{SCF}, \mathrm{CBF}$ - in 8 .

- VF, APF - in 9 .

- $\mathrm{SCF}, \mathrm{NNF}$ (two) - in [10].

- $\mathrm{CF}$ - in [11.

- $\mathrm{BF}$ - in [12].

- Where:

- STF - Sierpinski triangle (gasket) fractal.

- SCF - Sierpinski carpet fractal.

- VF - Vicsek fractal.

- BF, BFm - Box fractal (and modifications).

2010 Mathematics Subject Classification. Primary: 15A69 Secondary: 28A80, 37F45. 
- CF - Carpet fractal (like Sierpinski's, but asymmetric).

- HGF - Hexagon fractal.

- CBF - Checkerboard fractal.

- APF, APFm - "Airplains" fractal (and modifications).

- XDF - Diagonal cross fractal.

- NNF - "No Name" fractal (new one, but not named yet).

In this article it will be shown that only three prime classes (described below), i.e., "A", "O" and "X" (representing accordingly: triangular, square/rectangular and cross patterns) are able to define and replicate well-known popular fractals like Sierpinski triangle [13, 14] and carpet [15, 16], also Vicsek [17] and Box [18] fractals. Not to mention many other popular fractals not named yet, but, at least, presented with images in some resources. In addition, demonstrating many nice derivatives of these 3 basic classes, and defining many new classes able to produce both the same and thousands of the new patterns. Also, it will be shown why these mentioned above fractals were discovered and became highly referenced and popular. Finally, this classification paves the way to discover on the regular basis thousands of the new fractals, and this gives researchers a wider range of fractal models to choose from.

\section{Definitions}

Let's start with a few definitions used for classification purposes here. Note: these definitions are related and applied only to matrices used for fractal images generation using the Kronecker power (based upon the self Kronecker product).

Definition 2.1. The Kronecker power $n$ of a matrix $M$ is defined as $M \otimes M \otimes \ldots \otimes M$, i.e., $n$ times self Kronecker product. In short: $M^{n \otimes}$.

\section{Remarks:}

- From now on the term Kronecker power based fractals will be used, because it is more precise.

- It is often said that matrix $M$ has an order (or level) $n$ if it has the Kronecker power $n$. Same order is used for the generated fractal image, related to matrix $M$.

Note: a matrix declared an "initial" has the order 1 , even if it is, in fact, a resultant order $N$ matrix generated previously.

Here is another important definition:

Definition 2.2. A matrix containing zeros and ones will be called the fractal matrix if it has at least one zero and one number one.

Some initial matrices (even fractal ones) can't, produce fractals. So, here is a definition for them:

Definition 2.3. A matrix is called degraded if it contains all zeros or only one number one.

A little bit expanded definition of the degraded matrix is the following:

Definition 2.4. A fractal matrix that could not produce a fractal image is called degraded. 
Another useful definition is the following.

Definition 2.5. A fractal matrix is called inverted to another matrix if zero became one and one became zero.

The following statement/lemma could be easily checked and even proved formally:

Statement 2.6. The Kronecker product of a fractal matrix to any other fractal matrix is resulted in a fractal matrix, if the latter is a nonzero matrix.

Remarks:

- Here, usually, square matrices will be used, because they are producing symmetrical completed fractals in most cases. In general, the Kronecker power of a fractal matrix is producing mostly fractals.

- Rectangular matrices are important too, because in some cases they allow to eliminate extra margins.

\section{Demonstrating Classifications of Selected Classes}

3.1. Class notation. A class notation is very simple, i.e., a capital letter (defining class) followed by an optional small letters (indicating a subclass, a type, etc.), next are numbers representing initial matrix sizes (mostly one number for the square size), next - a small letter 'o' (reserved for an order) and it follows by a number (an order). Small letters ' $m$ ', 'i', 'f' and ' $x$ ' are reserved for the types "modified", "inverted", "framed" and "multiplied" accordingly.

So, in other words, a class notation is describing the following properties:

- A class pattern - a capital letter pointed to it (e.g., an "A" reminds you about the triangular pattern);

- Next could be an optional small letter (indicating subclass);

- Next could be a type 'm', 'i', 'f', 'x', etc.;

- Next - basic square initial matrix size, e.g., 3 means that a matrix is $3 \mathrm{x}$ 3 (we have a notation 'A3' for now, and it's enough to describe the initial matrix);

- Next could be 'o4' - means that a final matrix will be having an order 4 , or $3^{4} \times 3^{4}$ in size (as result, we have the 'A3o4' for the final fractal matrix);

- Note: other modifiers possible, and the listed ones could be placed in other positions if it is still understandable.

A "scheme" is actually a graphical representation of the initial fractal matrix.

Note: in the images of "class schemes" - numbers 1 in all initial matrices are shown as a big rectangular navy colored dots. The gray color is used to indicate that this fractal matrix is already belonging to another class, but its pattern is used for the current class.

3.2. "A" class (triangular pattern) and its samples. It should be mentioned here, that there is only one valuable A2 fractal matrix, because all others are mostly rotated $90^{\circ}$ copies of it or degraded. So, generated from them fractal images will be the same, but rotated, or no fractals at all. The same is true for all rotated copies of the initial matrices, i.e., they are the same from the point of view of this classification. 
In the Fig. 1 below find a few "A" class schemes.

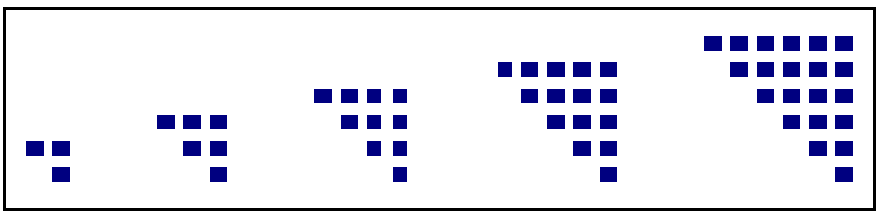

Figure 1. Schemes of the "A" class (A2-A6)

The Fig. 2 below shows the Grandfather fractal of the "A" class and two Triangle sibling fractals (A3, A4).
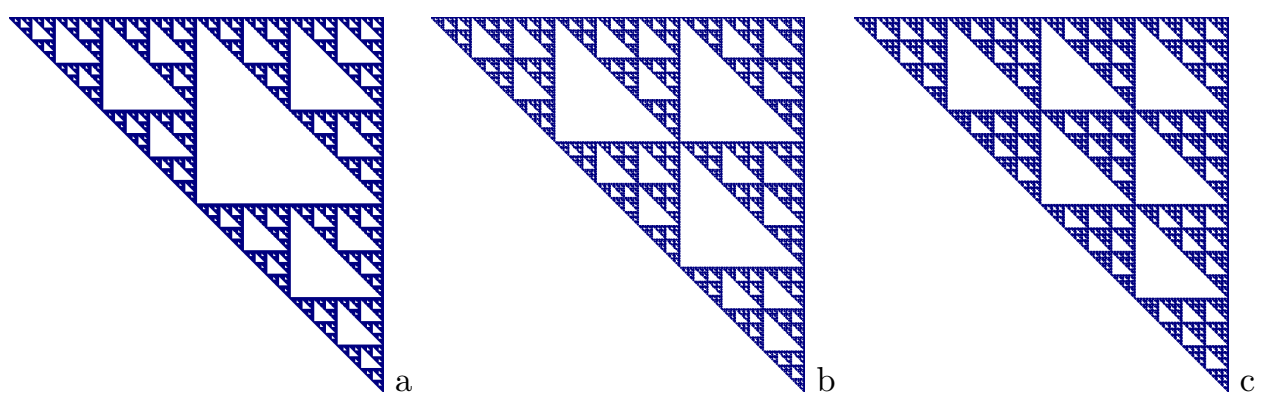

FiguRE 2. a) Sierpinski triangle fractal (A2o9); b) Triangle A3o5 fractal; c) Triangle A4o5 fractal.

The Fig. 3 below shows two Triangle sibling fractals of the "A" class (A5, A6).

Note: A2-A6 aka "Pascal triangle modulo 2-6". See [19, 20].
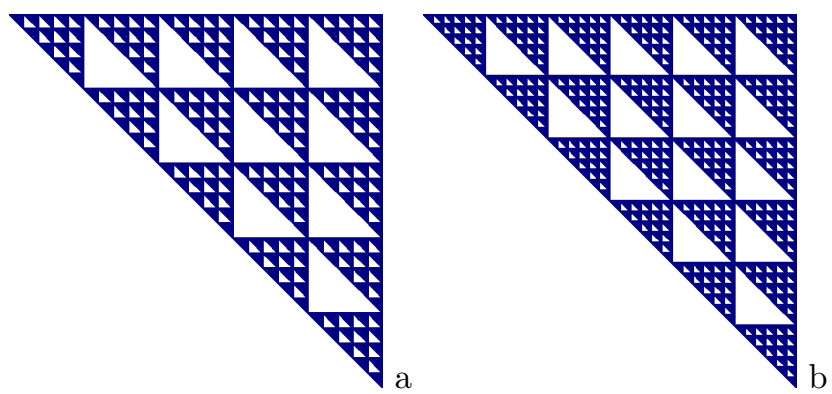

Figure 3. a) Triangle A5o4 fractal; b) Triangle A6o4 fractal.

3.3. "Aa" - "Ad" subclasses and their samples. "Aa" - "Ad" subclasses are, actually, different modifications of the "A" class.

In the Fig. 4 below find a few "Aa" - "Ad" subclasses schemes. In the Fig. 5 below find a few "Ac", "Ad" subclasses schemes.

The Fig. 6 presents selected Triangle sibling fractals of the "Aa" - "Ad" subclasses: 


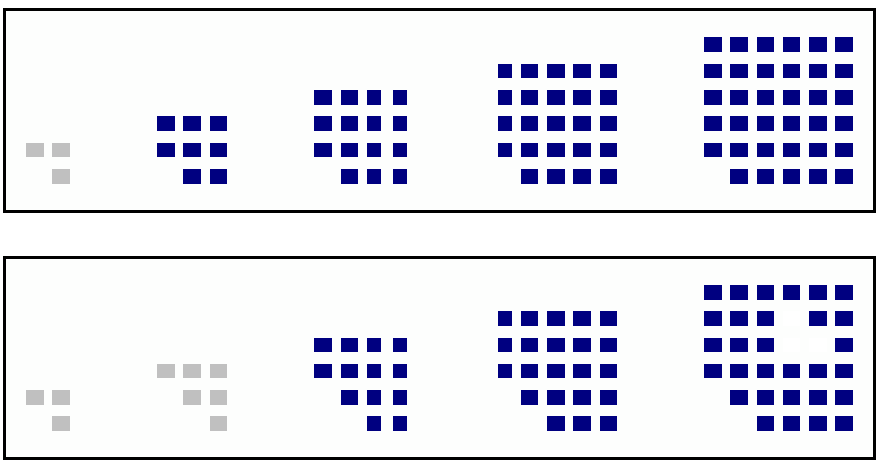

Figure 4. Schemes of the "Aa3" - "Aa6"

and the "Ab4" - "Abm6" subclasses

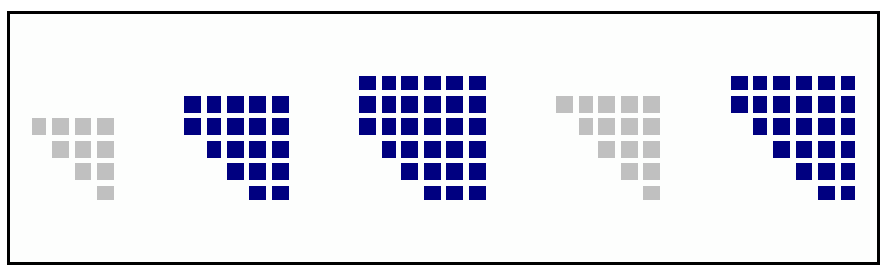

Figure 5. Schemes of the "Ac5", "Ac6", and "Ad6" subclasses
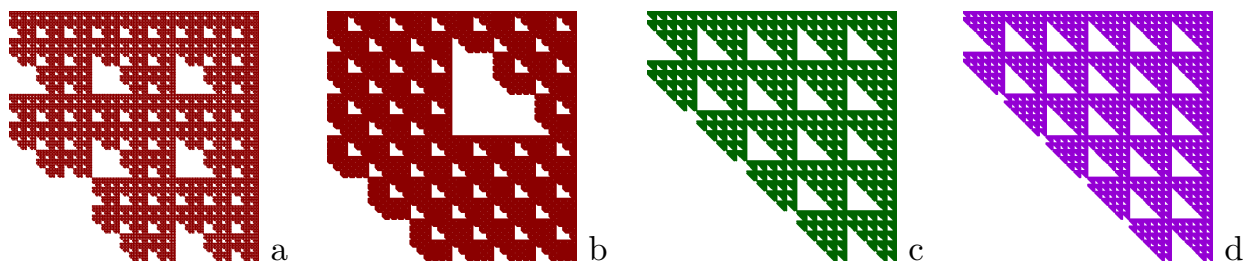

Figure 6. Triangle sibling fractals: a) Aa3o5; b) Abm6o4; c) Ac5o4; d) Ad6o4.

3.4. "O" class family samples (square/rectangular pattern). In the Fig. 7 find a few "O" class schemes.

Note: to make it more interesting the number 1 was put in the center of O7 initial matrix, so, now it is modified Om7 fractal matrix.

The Fig. 8 below presents the Grandfather fractal of the "O" class:

The figures below are Rug sibling fractals of the "O" class:

3.5. "O3xN" class (multiplied $N^{2}$ times $\mathrm{O} 3$ matrices) and its samples. This class represents multiplied/repeated $N^{2}$ times O3 matrix with partially overlapped edges. It's demonstrated here, because it's producing square/rectangular patterns.

The sub-figures of Fig. 11 below are Rug sibling fractals of the "O3x" class:

3.6. "MN" class (size $\mathrm{N}$ matrix with any pattern) and its samples. This class represents matrices of the size $\mathrm{N}$ with any weak or undefined pattern (schemes 


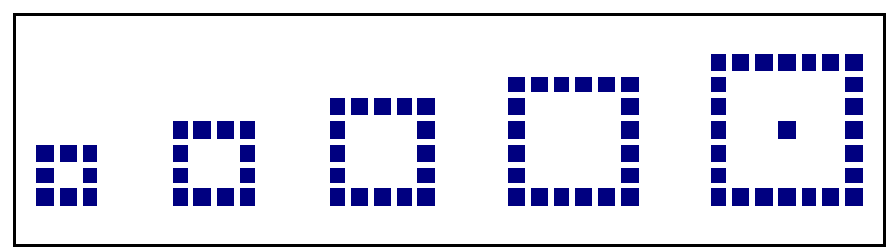

Figure 7. Schemes of the "O" class (O3-Om7)

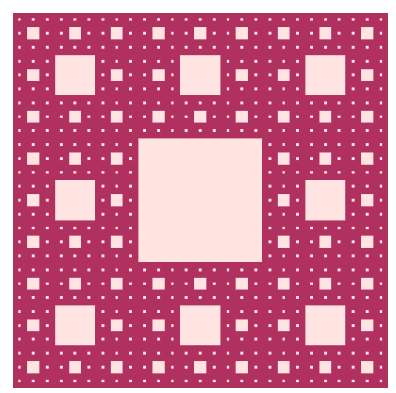

Figure 8. Sierpinski carpet fractal, order 6 (O3o6)
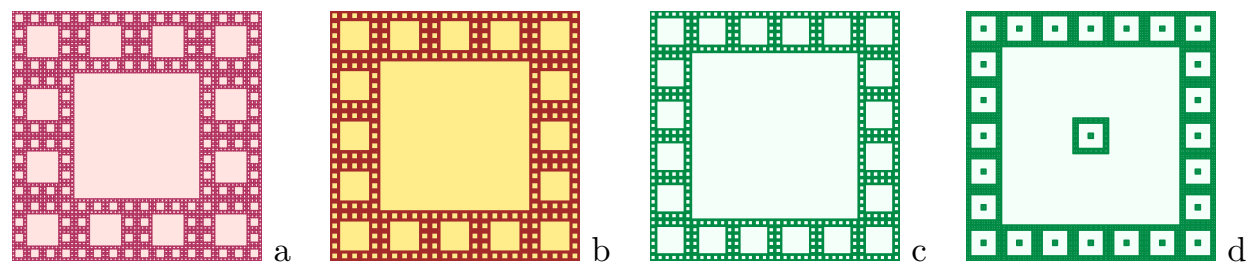

Figure 9. Rug fractals: a) O4o4; b) O5o4; c) O6o4; d) Om7o4.

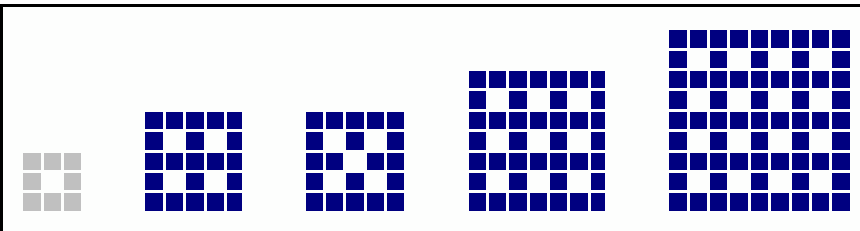

Figure 10. Schemes of the "O3xN" class (O3x2, O3xm2, O3x3, O3x4.)

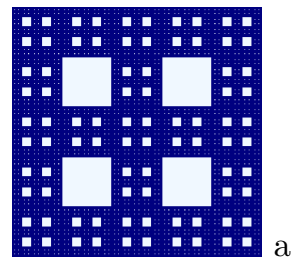

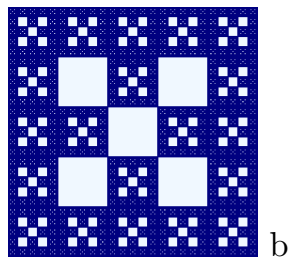
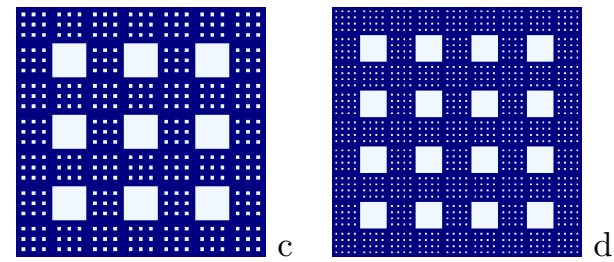

Figure 11. Rug fractals: a) O3x2o4; b) O3xm2o4; c) O3x3o4; d) O3x4o3. 
in Fig. 12). It's demonstrated here, because it's producing square/rectangular patterns.

Also, it is useful to describe all possible fractal matrices of the size 2, for example. Note: only M2 (equal to A2) is a valuable fractal matrix. See "A" class above. Find selected schemes of the "MN" class below.

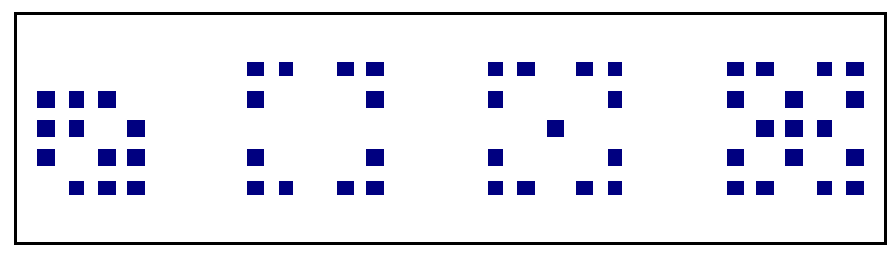

Figure 12. Schemes of the "MN" class (M4 'diagonal', three M5 'rhombuses')

The sub-figures of Fig. 13 below are Rug sibling fractals of the "MN" class.
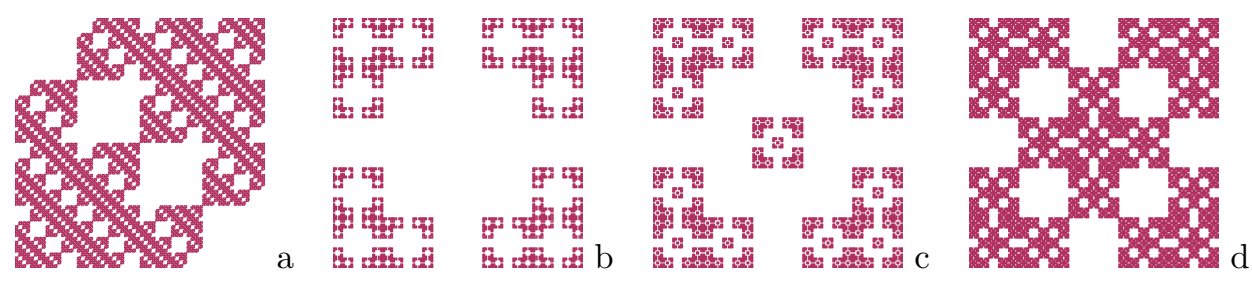

Figure 13. Rug fractals: a) M4do4; b) M5ro4; c) M5ro4a; d) M5ro4b.

3.7. "X", "Xd" class samples (upright and diagonal cross patterns).

The Fig. 14 below shows the Grandfather's fractals of the "X", "Xd" classes (schemes are in Fig. 15):
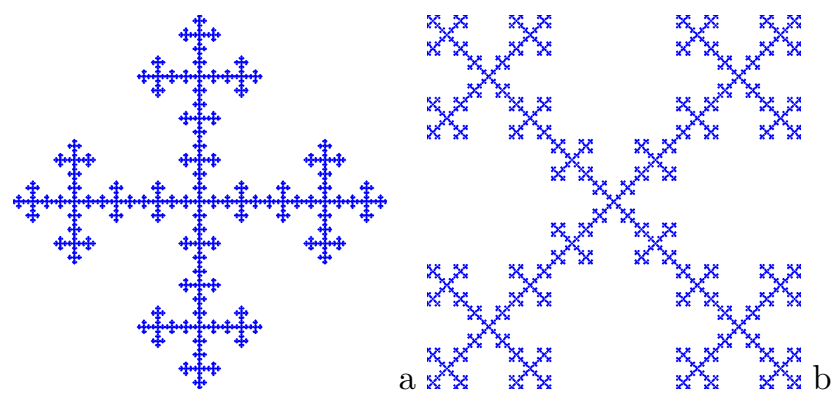

Figure 14. a) Vicsek fractal (X3o5); b) Box fractal (Xd3o5).

The Fig. 16 below shows Cross sibling fractals of the "X", "Xd" classes:

Note: the cross sibling Xd4o4 fractal (Fig. 16c) is a pretty popular too. It was shown in a few sources.

Another interesting class is "Framed inverted $\boldsymbol{X} \boldsymbol{d}$ class". Or FiXd for short. In our case: 


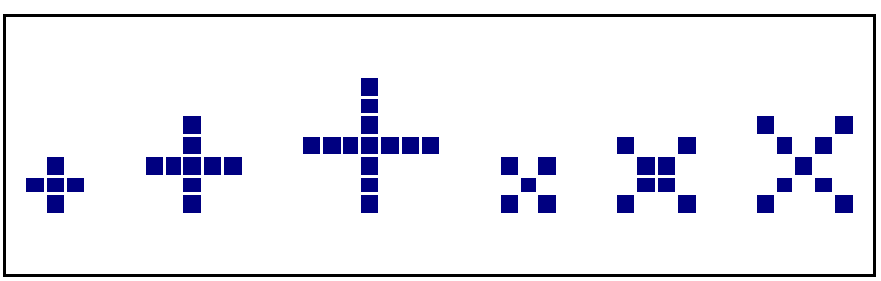

Figure 15. Schemes of the "X", "Xd" classes
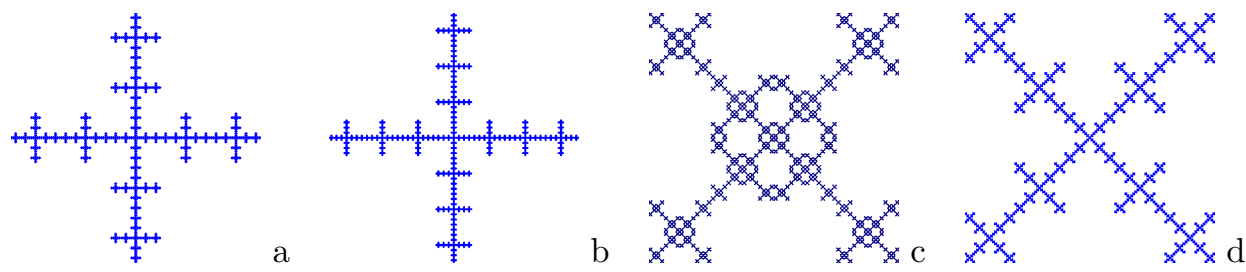

Figure 16. Cross fractals: a) X5o4; b) X7o4; c) Xd4o4; d) Xd5o4.

- Framed means that Xd matrix is put inside frame consistent of only 'one' numbers.

- Inverted means that Xd matrix was inverted, i.e., zero became one and one became zero. Note, inversion is better before framing.

The Fig. 17 below shows two the letter ' $\mathrm{X}$ ' fractals of the "FiXd" class:
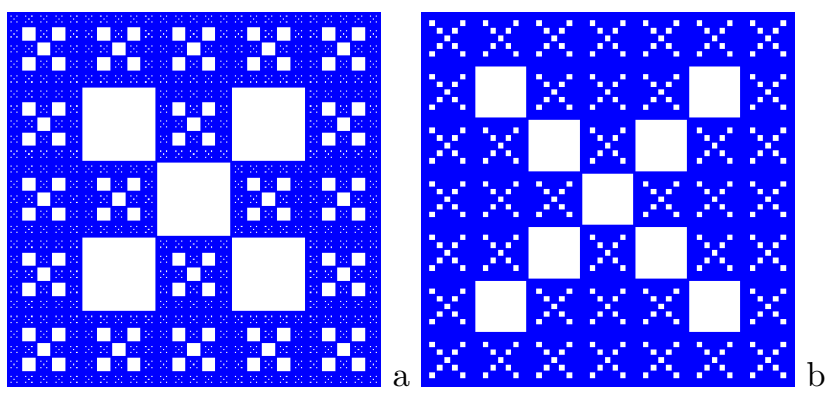

Figure 17. Letter 'X' fractals: a) FiXd3o4; b) FiXd5o4.

At the same time, these 2 fractals could be called "Oz" fractals producing square pattern, for example.

Sub-classes "Xw", "Xu" (see schemes in Fig. 18) represent, accordingly, swastika and sauwastika [21, 22]. I bet, - you never seen such fractals before.

The images that these fractals are representing, for sure, are not popular, but, actually, they are ancient signs misused in the recent history.

Note: "Xw" and "Xu" sub-classes have similar just mirrored images.

In the Fig. 19 below find two Cross sibling fractals of the "Xu" sub-class. 


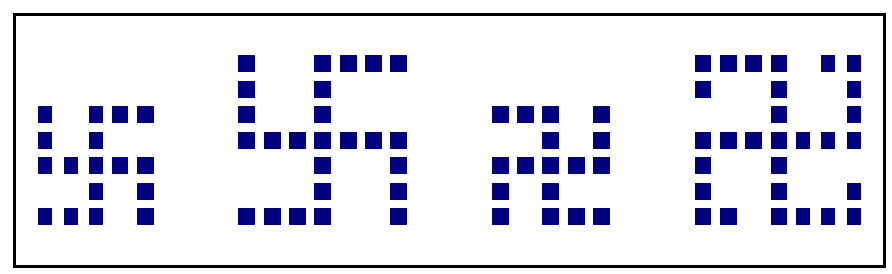

Figure 18. Schemes of the "Xw", "Xu" sub-classes

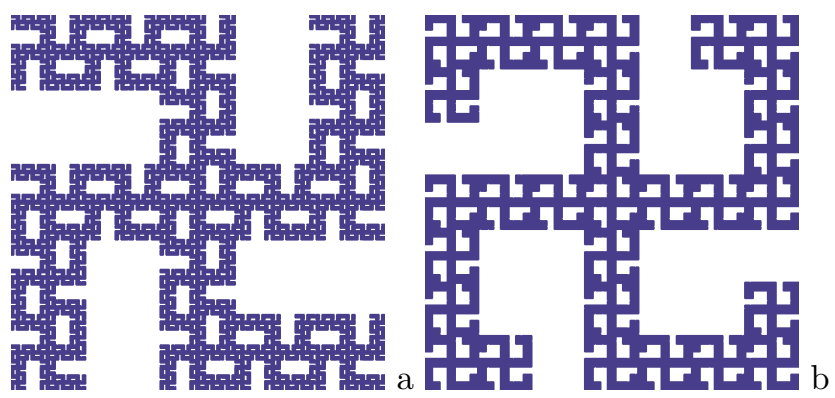

Figure 19. Cross fractals: a) Xu5o4; b) Xum7o4.

3.8. "F" class (framing other class) and its samples. Note: the schemes of only two framed classes "A" and "Ab" are demonstrating in Fig. 20, to give you an idea of the framing.

The meaning of "frame" was already explained above, and samples of a few fractal images were shown.

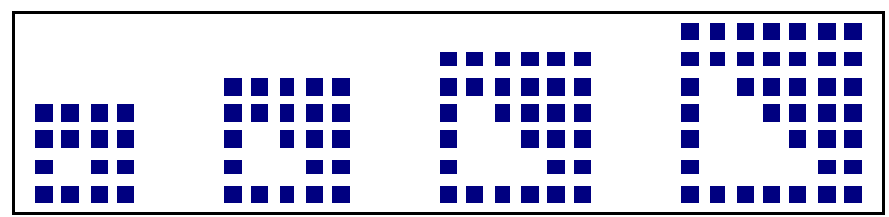

FiguRE 20. Framed "A" and "Ab" class (FA2, FAb3 - FAb5)

3.9. " $Q$ " and " $R$ " classes (quasi-random and random classes) and their samples. Remarks:

- "Q" class simply means that having matrix filled with only number 1, zeros are added according to some sort of algorithm (including the one in your mind). See Fig. 21a. The same could be applied to the inverted matrix (i.e., initially filled with zeros).

- "R" class simply means that having matrix filled with number 1 , - zeros are added using random algorithm of placing. See Fig. 21b. The same could be applied to the inverted matrix. 


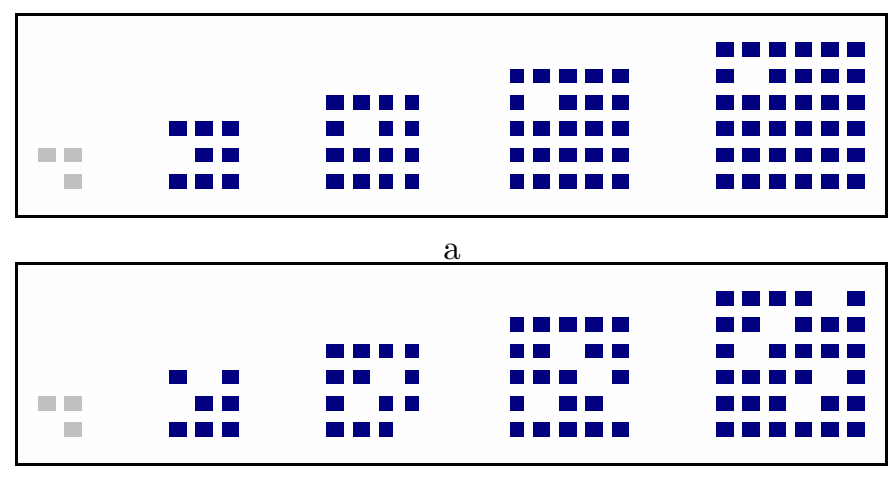

$\mathrm{b}$

Figure 21. Selected schemes of the "Q" and "R" classes: a) Q3Q6; b) R3-R6.

3.10. "L" class samples (letters, numbers and signs). The idea of "L" class has sprung up after discovering FiXd5 fractal (shown above). So, all matrices in "L" class are "framed" and "inverted" matrices.

In [2, 3, 23] already were demonstrated such letter, number and sign fractals as following: 'H', 'T', 'AV', '5', '6' and '+ sign' fractals. That's why just a few new "L" class fractals are shown below (schemes are in Fig. 22).

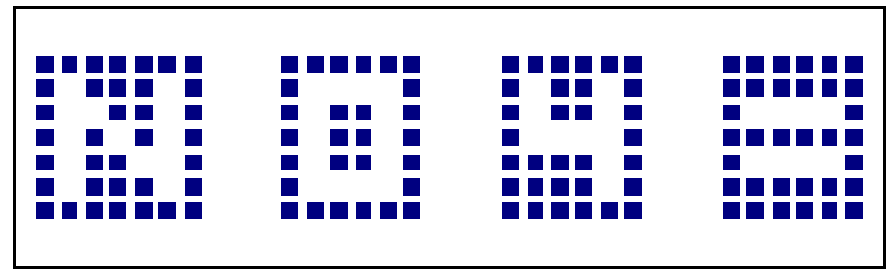

FiguRE 22. "L" class selected schemes (LN7, LO7, LN47, LEs7)

The Fig. 23 below shows letters, number and sign fractals of the "L" class:
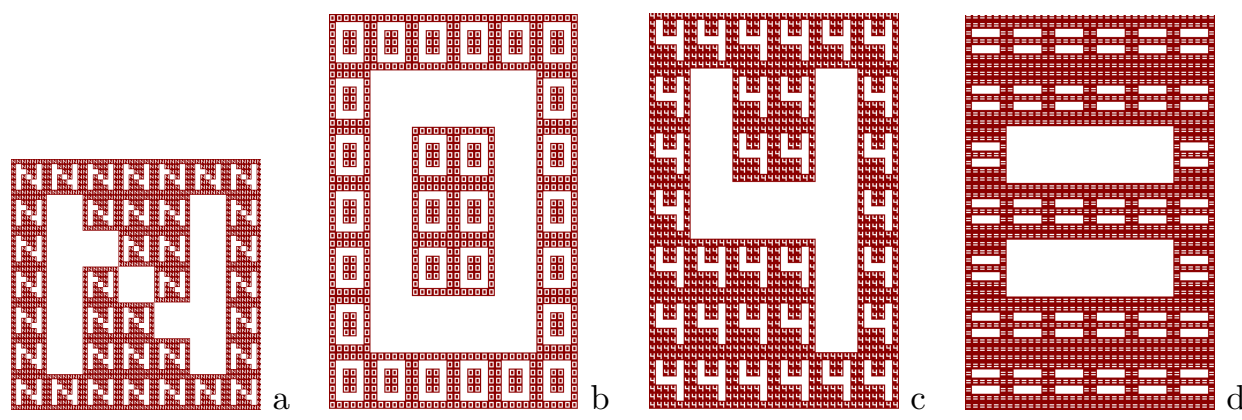

Figure 23. Fractals: a) Letter 'N' (LN7o3); b) Letter 'O'

(LO7o3); c) Number '4' (LN47o3); d) Equal sign (LEs7o3). 


\section{Conclusion}

I'm sure, the most important fact was already noticed, i.e.,

All discovered highly popular fractals are derived from minimal matrices of the 4 classes: A2, О3, X3, Xd3 and Xd4.

Minimal fractal matrix means minimal number of dots and ease to construct 2nd - 4th orders. That's why they were discovered, that's why they have so many "constructing" approaches [14, 16, 17, 18, including interacting ones, also primitive, like cutting and folding paper and so on. May be, because there was no an access to the powerful computer those days?

No doubt, the discovery of all these popular fractals was a very important step to push further researches.

At the same time, if an initial fractal matrix is a big one (e.g., M43),- would it be possible to "construct" related fractal? Definitely not. Even not so big (just 25K dots) matrix O7m2o3 (see Fig. 24)) looks like not suitable for "constructing" without a computer. But look at it: isn't it a very nice and definitely the new type of rug fractal?

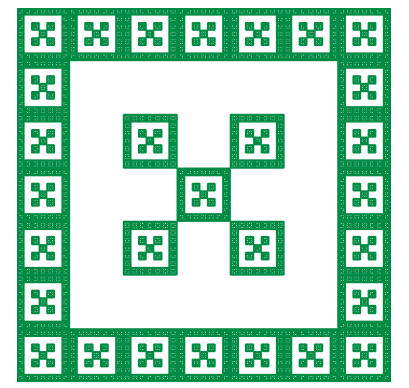

Figure 24. Rug O7m2o3 fractal

It was generated using the following initial matrix:

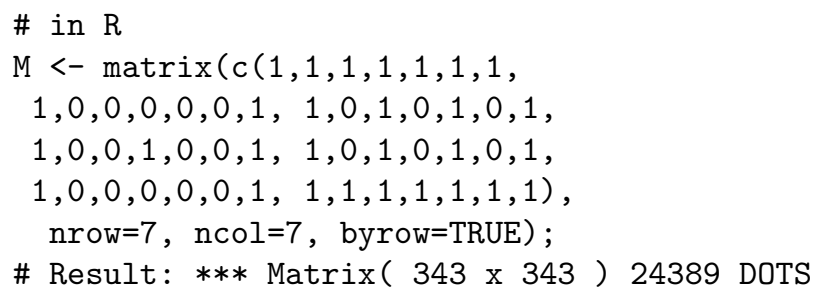

It is interesting and remains as an open question: Why using different approaches, e.g., Kronecker power based, IFS based [3, 24, L-system generated 25, [26], etc., or even using just a recursive function [13, 26, 27, - it is still possible to reproduce Sierpinski carpet or triangle, for example? See samples below.

I want to stress another analogy that looks like the true one for me.

Let's start with the following fact: nobody yet invented a formula or a function for prime numbers. Instead, researches are using the Zeta function and many alike functions to discover distribution properties of prime numbers. 

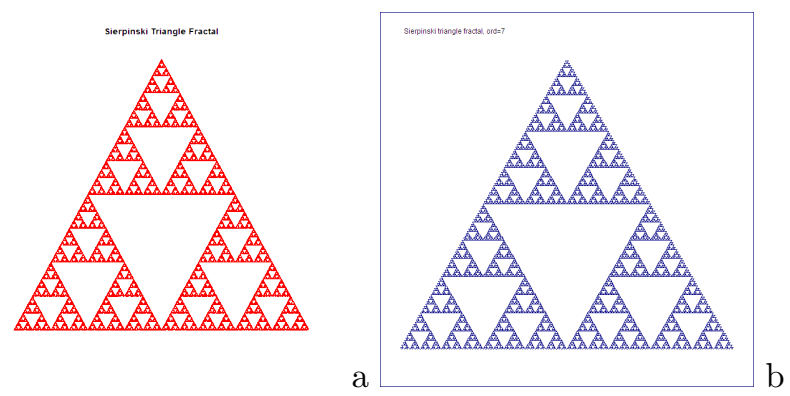

FigURE 25. a) Sierpinski triangle (IFS generated); b) Sierpinski triangle (L-system generated)

Here is the conjecture:

The Kronecker power is the prime function to discover many unique replication and other properties of many fractals. Even if they can't be generated by this function.

One related to this conjecture example discussed in [19. It shows that although generated KPBF "Hexagon" is looking like distorted "Hexaflake" 20] they both have an equal Hausdorff dimensions. Let's say that KPBF "Hexagon" is a simulation of the "Hexaflake".

So, it is possible to simulate a fractal (plotted using different method) and calculate its Hausdorff dimension. May be, it would be only the approximation of its dimension, but still it is a very useful property for analysis.

It should be also emphasized, that all shown above class schemes could be expanded to represent bigger matrices. In such case, for the big initial matrix plotting, - any user needs a very powerful computer, also a big canvas and a scaling applied to the final fractal image, especially, if the one wants to make visible all orders.

Finally, this classification definitely paves the way to discover thousands of the new fractals. In addition, it was already demonstrated how to build new scheme (aka initial fractal matrix) for any new desired fractal model. Moreover, everyone has simple, flexible, fast and ready to use "Design" / "Plot" pages [2] able to produce new fractals instantly. A number of the new fractal patterns is practically unlimited.

\section{REFERENCES}

[1] W.-H. Steeb. Problems and Solutions in Introductory and Advanced Matrix Calculus, pages 152-173. World Scientific Publishing, 2006. ISBN: 981-256-916-2.

[2] A.E. Voevudko. Generating kronecker product based fractals. Code Project, 2017. https://www.codeproject.com/Articles/1189288/Generating-Kronecker-Product-BasedFractals.

[3] A.E. Voevudko. A few approaches to generating fractal images in r. Code Project, 2017. https://www.codeproject.com/Articles/ 1195034/A-Few-Approaches-to-Generating-FractalImages-in-R.

[4] M.J. Gazalé. Gnomon: From Pharaohs to Fractals. Mathematics, Princeton University Press, Princeton (N.J.), 1999.

[5] Hardy A. and W.-H. Steeb. Mathematical Tools in Computer Graphics with C\# Implementations. World Scientific Publishing Company Pte Limited, 2008. ISBN: 9789812791023. 
[6] Leskovec J., Chakrabarti D., and Kleinberg J. at al. Kronecker graphs: An approach to modeling networks. Journal of Machine Learning Research, 11, 2010. https://cs.stanford.edu/people/jure/pubs/kronecker-jmlr10.pdf.

[7] A. Charpentier. Fractals and kronecker product. Freakonometrics, 2012. http://freakonometrics.hypotheses.org/2474.

[8] W.-H. Steeb. The Nonlinear Workbook: Chaos, Fractals, Cellular Automata, Genetic Algorithms, Gene Expression Programming, Support Vector Machine, Wavelets, Hidden Markov Models, Fuzzy Logic with C++, Java and Symbolic C++ Programs Sixth Edition. World Scientific Publishing Company Pte Limited, 2014. ISBN: 9789814583497.

[9] R. Wicklin. Self-similar structures from kronecker products. 2014. http://blogs.sas.com/content/iml/2014/12/17/self-similar-structures-from-kroneckerproducts.html.

[10] S.V. Petoukhov. Music and the modeling approach to genetic systems of biological resonances (genetic system and vibrational mechanics). International conference IS $4 I S, \quad V i$ enna, Austria, 2015. http://petoukhov.com/wp-content/uploads/2011/05/sciforum-003888from-PETOUKHOV-2.pdf.

[11] Stepanyan I.V. and Petoukhov S.V. The matrix method of representation, analysis and classification of long genetic sequences. Information, MDPI, 8(12), 2017. doi: 10.3390/info8010012.

[12] Karagiannakis P. and Weiss S. Analysis of a purina fractal beamformer. Conference on Signals, Systems and Computers, 2017. doi: 10.1109/ACSSC.2013.6810320.

[13] RosettaCode. Sierpinski triangle, 2015. http://rosettacode.org/wiki/Sierpinski triangle/.

[14] Wikipedia. Sierpinski triangle. https://en.wikipedia.org/wiki/Sierpinski_triangle.

[15] RosettaCode. Sierpinski carpet, 2015. http://rosettacode.org/wiki/Sierpinski carpet/.

[16] Wikipedia. Sierpinski carpet. https://en.wikipedia.org /wiki/Sierpinski_carpet.

[17] Wikipedia. Vicsek fractal. https://en.wikipedia.org/wiki/Vicsek_fractal.

[18] Math World. Box fractal. http://mathworld.wolfram.com/BoxFractal.html.

[19] A.E. Voevudko. Fractal dimension of the kronecker product based fractals. 2018. arXiv:1803.02766 [math.DS].

[20] Wikipedia. List of fractals by Hausdorff dimension. https://en.wikipedia.org/wiki/List_of_ fractals_by_Hausdorff_dimension.

[21] Wikipedia. Swastika. https://en.wikipedia.org/wiki/Swastika.

[22] Encyclopaedia Britannica. Swastika. https://www.britannica.com/topic/swastika.

[23] RosettaCode. Kronecker product based fractals, 2017. http://rosettacode.org/wiki/ Kronecker_product_based_fractals.

[24] Wikipedia. Iterated function system. https://en.wikipedia.org/wiki/Iterated_function_ system.

[25] Wikipedia. L-system. https://en.wikipedia.org/wiki/L-system.

[26] A.E. Voevudko. Ability of the L-system in $\mathrm{R}$ comparing to well-known tools. Code Project, 2017. https://www.codeproject.com/Articles/1195841/Ability-of-the-L-system-inR-Comparing-to-Well-kno.

[27] Wikipedia. Chaos game. https://en.wikipedia.org/wiki/Chaos_game.

CSUlB - California State University, Long Beach, CA ... November 3, 2017

E-mail address: AnatolV@outlook.com 